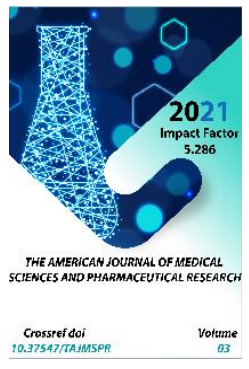

\title{
Gas Analytic Method For Diagnostics Of Ischemic Heart Disease
}

Gulchekhrakhon Ibrakhimovna Qodirova

Associate Professor, Candidate Of Medical Sciences, Andijan State Medical Institute, Uzbekistan

\section{ABSTRACT}

The enormous damage to human health from cardiovascular disease (CVD) poses significant challenges to both clinicians and public health workers.

In most cases, CVDs develop latently; their clinical signs appear at a late stage. Often, patients are unaware of the presence of the disease and die suddenly; it is extremely rare for a doctor to have the opportunity to examine a patient before his cardiovascular system is seriously affected. It follows from this that therapeutic measures alone cannot solve the problem of death from CVD. There is a need to make significant changes in research methods, despite the fact that they are based on clinical methods

\section{KEYWORDS}

Exhaled air, ischemic heart disease, diethylamine, gas analysis, volatile biogenic amines.

\section{INTRODUCTION}

The purpose of the work is to study gas analytical methods and the choice of equipment for the diagnosis of coronary heart disease, the development of a method for taking samples of exhaled air and carrying out measurements and analysis of the results. 
The increased content of diethylamine in explosives is apparently associated with impaired metabolism of biogenic amines in NQMI. To identify the mechanism of disruption of the metabolism of biogenic amines, clinical observations should be carried out according to our method in the dynamics of the development and treatment of NQMI. The results obtained indicate the possibility of diagnostics based on the determination of the content of amines in the IV in patients with coronary artery disease. For early diagnosis of ischemic heart disease, a more detailed analysis of the amine content in explosives should be performed.

Early diagnosis of coronary heart disease (IHD) is an important task of modern cardiology. More than $70 \%$ of deaths in acute myocardial infarction (AMI) are observed in the first hours from the onset of the disease. It is known that the transamination enzyme, aspartate aminotransferase, significantly increases its activity in the period preceding AMI, i.e. in a pre-infarction state [3]. Recent studies indicate that to understand atherosclerosis and coronary heart disease, it is necessary to study biogenic amines (adrenaline, norepinephrine, serotonin, etc.) and their precursors, metabolic products and enzymes involved in their metabolism [2, 9, 11]. It was found that in patients with coronary artery disease, the activity of monoamine oxidases (MAO) is reduced by 2 times, and in acute myocardial infarction by 2.5 times [8]. Modern methods of enzymatic analysis, although they can reliably determine the preinfarction state, nevertheless, their use is limited for the following reasons: 1 - The invasiveness of the methods used; 2 - Non-expressive diagnosis of coronary artery disease. That is why at present the method of analysis of exhaled air (IV) is of great importance as the most promising express method of non-invasive technologies for diagnosing coronary artery disease [4].

\section{MATERIAL AND METHODS}

To obtain a BB sample in a hospital, 78 surveyed men aged 20-64 years were divided into the following 3 groups: I (control) - healthy individuals aged 20-40 years - 20 people; II patients with coronary artery disease. Acute myocardial infarction without Q wave (NQMI) 25 people aged 32-59 years; Group III - patients with coronary artery disease. Postinfarction cardiosclerosis (PICS) with stable angina FC IIIV - 33 people aged 35-64 years. All patients were diagnosed on the basis of clinical observation data, laboratory analysis and functional diagnostics. For 3-15 years, patients underwent inpatient and outpatient treatment. Explosive samples were obtained using traps using bidistilled water in a volume of $200 \mathrm{ml}$ as an absorber. In the process of obtaining a hypoxemic IV sample, the condition of the patients was monitored with the help of an electronic device "Amulet-9" by ECG indicators (heart rate and ST interval). There was a slight acceleration of the heart rate (6-12 beats per minute). Signs of coronary insufficiency in the form of a decrease in the ST interval were not observed in any patient. The obtained samples of bidistilled water with explosives were poured into a special degasser with a thermal heater prior to analysis on an amine gas analyzer. The degasser temperature was $450 \mathrm{C}$. The gas flow with an admixture of volatile biogenic amines was directed to the surface-ionization sensor of the amines gas analyzer [5]. The flow rate (10 I / min.) And the pressure $(800 \mathrm{~mm} \mathrm{Hg})$ of the gas were controlled by an Argus spiro monitor.

Calibration of the amine gas analyzer. 
The amine gas analyzer was developed by specialists of the Institute of Electronics of the Academy of Sciences of the Republic of Uzbekistan, which allows detecting the amine content in the gas mixture up to 10-10 g / I [5]. Previously, we discovered the presence of diethylamine in IVs in patients with PICS [10], for which we calibrated this gas analyzer for diethylamine. For calibration, a $25 \%$ solution of nicotinic acid diethylamide was used. Acting on this drug with caustic soda and heating it, diethylamine was cleaved off. Passing diethylamine through bidistilled water, its various solutions were obtained with the

following concentrations: 10-4, 10-5, 10-6, 10-7, $10-8,10-9 \mathrm{~g} / \mathrm{l}$. The diethylamine solutions obtained in this way were used to calibrate the gas analyzer. The calibration factor for diethylamine is $\mathrm{K}=3 * 10-2$. The calculation of the concentration of diethylamine according to the readings of the gas analyzer was made according to the following formula: $\mathrm{C}=\mathrm{K} * \mathrm{~J}$, where $J$ is the readings of the gas analyzer ( $\mathrm{g} /$ I); $C$ is the concentration of diethylamine in the gas mixture $(\mathrm{g} / \mathrm{I})$.

RESULT AND DISCUSSION

Table 1.

\section{The content of diethylamine in the exhaled air in healthy and patients with coronary}

\section{heart disease.}

\begin{tabular}{|c|l|c|}
\hline № & & Amine content in explosives \\
Groups & Diagnoz & * $^{-9}$ г/л \\
\hline 1. & Health & $74 \pm 2$ \\
2. & NQMI & $879 \pm 261$ \\
3. & PEAKS. Stable angina. FC II-IV & $178 \pm 22$ \\
\hline
\end{tabular}

The table shows the mean values and the confidence interval of the amine content in explosives for all examined groups at $t=2$ according to the Student's criterion $(P<0.05)$. As can be seen from the table, the maximum level of amines in explosives is observed in group II $-879+261 * 10-9 \mathrm{~g} / \mathrm{I}$, which is $1087.8 \%$ higher than in the control group. In group III, there is a decrease in the content of amines to $178+22 * 10-9 \mathrm{~g} /$ I compared to group II and $140.5 \%$ more than in group I. Thus, the results obtained show that NQMI increases the yield of volatile amines in explosives. At the same time, the average value of diethylamine in myocardial infarction statistically significantly differs from the control $(t=3.084)$. However, the variance of the measured parameter differs significantly from the control at NQMI ( $\mathrm{mk}=2$; $\mathrm{mNQMI}=261$ ). The increased content of diethylamine in explosives is apparently associated with impaired metabolism of biogenic amines in NQMI. To identify the 
mechanism of disruption of the metabolism of biogenic amines, clinical observations should be carried out according to our method in the dynamics of the development and treatment of NQMI. The results obtained indicate the possibility of diagnostics based on the determination of the content of amines in the IV in patients with coronary artery disease. For early diagnosis of ischemic heart disease, a more detailed analysis of the amine content in explosives should be performed. It can be considered reasonable that the introduction of non-invasive diagnostics will bring significant benefits in the recognition of a number of difficult-to-diagnose diseases, as well as significantly protect and make the process of examining patients comfortable. IV analysis is a new direction in medical diagnostics. Despite the reliability of the pentane benchmark [7], its use in cardiology is associated with known difficulties in differentiating the disease. The proposed method for the diagnosis of ischemic heart disease by the content of volatile amines (especially diethylamine) in explosives does not have these disadvantages. The advantage of the method is complete harmlessness, expressiveness, the absence of the need for surgical intervention (implemented on an outpatient basis), the possibility of mass testing, an absolute guarantee of the impossibility of transferring the infection from the patient to the service personnel, and the low cost of the study. The difference observed in our work in the elimination of amines from IVs can become a reliable marker for early diagnosis of preinfarction state.

\section{CONCLUSIONS}

1. The amine gas analyzer, due to its high sensitivity, allows solving the problem of early diagnosis of coronary heart disease.

2. The content of amines in the exhaled air in patients with ischemic heart disease (especially in myocardial infarction) significantly increases in comparison with healthy people.

3. For the first time, a large dispersion in the yield of volatile amines at NQMI was found (due to the high sensitivity of the amine sensor), which is of interest in identifying the mechanism of the development of a heart attack.

\section{REFERENCES}

1. Askarov B., Khuzhamberdiev M.A. Simulation of living systems: theoretical analysis of metabolite metabolism of biogenic amines. Conference of Uzbekistan biochemists. 2006.17-18 p. 9.

2. Boimirzaev M.I., Khuzhamberdiev M.A. and other Dynamics of disorders and functions of the sympatho-adrenal system in patients with small focal myocardial infarction. Central Asian Medical Journal. 2002. No. 3-4, p. 227-230.

3. Mamadiev MM, Khuzhamberdiev MA, Askarov BA, Amanov K. Modeling of living systems: towards medical diagnostics based on physical methods of analysis of volatile metabolites of biogenic amines. 2019; 3-4: pp.187-189.

4. Meerson FZ .. Pathogenesis and prevention of stress and ischemic heart damage. Moscow: Medicine, 1984.

5. Rasulev U.Kh. and et all. Chromatographic determination of trece amounts of amines 
using a surfase ionization detector. Journal of Chromatography A. 704.1995.pp 473-482.

6. Klimenko VA, Krivorotko DN. Analysis of exhaled air as a marker of biochemical processes in the body. Medicine is theoretical. 2011; 1 (28): pp.138-143.

7. Phillips M. Analysis of exhaled air in medicine. In the world of science. 1992. No. 9-10. from. pp.42-48.

8. Kistenev YV, Chuikova KI, Gomboeva SS, Karapuzikov AA. Study of the relationship of absorption spectra of gas emissions from patients with acute viral hepatitis, obtained by laser optical-acoustic spectroscopy, with biochemical blood parameters. Honey. physics. 2017; 4: pp.3237.

9. What the disease smells like. Feldsher. Ambulance information site; http://www.feldsher.ru/news-view1258.html.

10. Khuzhamberdiev MA, Askarov B. Methodology for analyzing exhaled air as a possible criterion in the diagnosis of cardiovascular diseases. Journal of Theoretical and Clinical Medicine. 2012.No. 1, pp. 114-117.

11. Lavoisier Antoine Laurent. Science and technology section: Chemistry / Around the World. Online encyclopedia;

12. http://www.krugosvet.ru/enc/nauka_i_te hnika/ himiya LAVUAZE_ANTUAN_LORAN. 